\title{
Experimental Model for a Seismic Landmine Detection System
}

\author{
Waymond R. Scott, Jr., Member, IEEE, James S. Martin, and Gregg D. Larson
}

\begin{abstract}
A laboratory-scale experimental model has been developed and tested for a system that uses artificially generated high-frequency seismic waves in conjunction with a radar-based noncontact displacement sensor to detect buried landmines. The principle of operation of the system is to measure the transient displacement field very close to a mine location. In this way, the absorption and the geometrical spreading of the seismic waves have not reduced the effects of the mine. By using a seismic excitation, the system exploits the large difference between the elastic properties of a mine and the surrounding soil. This difference causes seismic wave interactions in the vicinity of a mine to be quite distinctive and provides a method for imaging mines and distinguishing them from typical buried clutter. Images of a variety of simulated and inert anti-tank and anti-personnel mines have been formed using this system. Burial scenarios involving natural clutter (rocks and sticks), light surface vegetation, localized burial effects, and multiple mines in close proximity have been studied. None of these scenarios appears to pose serious problems for detection performance.
\end{abstract}

Index Terms-Acoustic, elastic waves, landmine, radar, seismic.

\section{INTRODUCTION}

$\mathbf{S}$ EISMIC techniques show considerable promise for the reliable detection of all types of buried mines, even low-metal anti-personnel mines. The reason for this is that mines have mechanical properties that are significantly different from soils and typical forms of clutter. For example, the shear wave velocity is approximately 20 times higher in the explosive and the plastics used in typical mines than in the surrounding soil [1]. In addition, mines are complex mechanical structures with a flexible case, a trigger assembly, air pockets, etc. This complex structure gives rise to structural resonances, nonlinear interactions, and other phenomena that are atypical for both naturally occurring and man-made forms of clutter. This phenomenology can be used to distinguish a mine from clutter.

The range of burial depths typically associated with anti-personnel (AP) and anti-tank (AT) mines is from flush with the earth's surface to a depth of 10 to $20 \mathrm{~cm}$. AT mines are typically larger and more deeply buried than AP mines. These characteristics make classical seismic techniques ill suited

Manuscript received June 8, 2000; revised October 30, 2000. This work was supported in part by the OSD MURI Program and by the U.S. Army Research Office under Contract DAAH04-96-1-0448.

W. R. Scott, Jr. is with the School of Electrical and Computer Engineering, Georgia Institute of Technology, Atlanta, GA 30332 USA (e-mail: waymond.scott@ece.gatech.edu).

J. S. Martin and G. D. Larson are with the School of Mechanical Engineering, Georgia Institute of Technology, Atlanta, GA 30332 USA (e-mail: james.martin@me.gatech.edu; gregg.larson@me.gatech.edu).

Publisher Item Identifier S 0196-2892(01)05107-5. to the landmine detection problem. Seismic surveys with ground contacting geophones are intended to detect targets that are much larger and more deeply buried than landmines. These techniques usually involve the generation and detection of bulk waves (shear or compressional waves) in the earth. Surface guided (Rayleigh) waves are problematic for classical seismic measurements because their displacements decay exponentially away from the Earth's surface. They do not interrogate soil deeper than their wavelength, and they have relatively large surface displacements that can obscure other signals of interest. These features make the Rayleigh wave an excellent interrogation signal for the detection of land mines. Here, a signal with an appropriate frequency content will interrogate only the burial range of the targets of interest. Fig. 1 shows the wave fronts generated by an impulsive point source on an isotropic, homogenous elastic half space computed numerically [2]. The numerical computation was performed using a three-dimensional (3-D) finite-difference time-domain model. A free surface boundary condition is used to model the boundary between the air and the earth. A differentiated Gaussian pulse with a center frequency of $450 \mathrm{~Hz}$ is launched at $t=0$ from a velocity source on the surface. The magnitude of the vertical component of velocity is plotted at $t=10 \mathrm{~ms}$. The region plotted is a $3 \mathrm{~m} \times 6 \mathrm{~m}$ cross-section through the ground. The incident signal, length scales, and time instant plotted have been selected so that the wavefronts have separated in space and correspond to parameters of the experimental system. The color map indicated in the figure is used throughout this paper, with the scales indicated in the figures. The Rayleigh wave and the bulk compressional and shear waves are indicated. The Rayleigh wave front can be seen to penetrate to a shallower depth and to be stronger near the free surface. This is the region in which mines are buried.

Previous attempts to detect buried mines by surface wave scattering have been confounded by the small size of mines and the high attenuation of most soils [3]. These force competing requirements for a classical, monostatic pulse-echo detection system: low frequencies are required to propagate measurable energy over the two-way path from the source to the mine location and back to the receiver while high frequencies are required for a mine to scatter waves of appreciable strength. This observation leads several authors to suggest that a noncontact sensor could be used to measure seismic motion at the mine [3], [4]. Such a sensor is at the heart of the system currently under investigation as it offers three distinct advantages over a remote-receiving array. First, it obviates the need for the scattered wave to propagate back to a receiver location in order to achieve detection, thus eliminating one half of the geometric spreading 


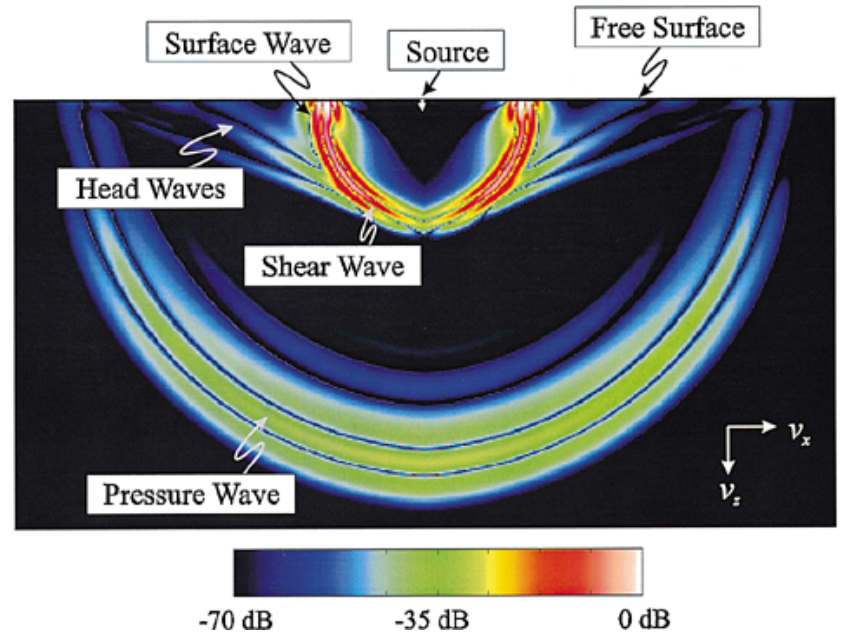

Fig. 1. Waves generated by a point source in an elastic half space.

and attenuation. Second, it removes ambiguities that might arise over the location of a target with a sparse remote array of receivers and/or a complicated propagation path. Third, it allows the measurement of nonpropagating motion above buried mines that may be detected only in the seismic near field of a mine.

All of the experiments reported in this paper have shown the existence of significant nonpropagating motion above buried mines. This is not surprising since nonpropagating wave-field components are a ubiquitous feature of near-field scattering and radiation problems. The most commonly cited examples of these are subsonic (evanescent) wavenumbers present on and near the surface of acoustically large objects. However, nonradiating components arise in extremely simple problems involving small sources also. One example of this would be the field produced by a radially oscillating sphere in an unbounded fluid. In addition to the propagating velocity component that is in phase with the acoustic pressure and has $1 / r$ geometric spreading, there is a second, nonradiating, component that is in quadrature with the pressure and spreads as $1 / r^{2}$. If the sphere under consideration is acoustically small $(k a \ll 1)$, then the velocity close to the object is dominated by this nonradiating component [5].

Other investigators have used a laser-based local displacement sensor in conjunction with uniform, continuous aero-acoustic excitation of the soil surface for mine detection [6]. Although this method appears to be similar to the one presented here, the underlying physical principles are quite different for the two methods. In the aero-acoustic method, the seismic interrogation signal was comprised of compressional waves propagating into the porous surface soil layer. The primary detection cue was a change in the local input impedance. This change was attributed to the significant differences in the porosity of the soil and the mine. In the seismic method presented here, the primary detection mechanism is motion of the mine excited by its interaction with the Rayleigh wave. The spatial/temporal imaging scheme used here is tied to the nature of a transient incident signal which propagates in the measurement plane; this would not be possible for the downwardly propagating continuous excitation.
Both Rayleigh waves and air coupled pore compressional waves decay with depth into the soil. For a Rayleigh wave, penetration depth is related to the wavelength measured along the surface. However, the penetration depth of pore compressional waves is a function of soil parameters that are not directly accessible through surface displacement measurements. Thus, a Rayleigh wave incident signal offers the potential to exploit additional information concerning target depth.

\section{EXPERIMENTAL MODEL}

The mine detection system that has been modeled experimentally is depicted in Fig. 2. The system consists of a stationary seismic source and a moving radar sensor.

The source is an electrodynamic shaker that has been coupled to the ground by a narrow foot. This was designed by experimental iteration to preferentially generate Rayleigh waves. The foot, a thin rectangular aluminum bar approximately $25 \mathrm{~cm}$ long, was placed with the long dimension parallel to the $y$-axis as in Fig. 3. The Rayleigh wave generation appears to be dictated by the foot's perimeter and bulk wave generation by its surface area. Although this suggests that a knife edge geometry would be optimal, the foot must also carry a sufficient bias force (the weight of the shaker in this case) that contact with the surface is maintained. Without a sufficiently large contact area, the shaker foot will tend to bury itself.

The sensor, which measures the displacement of the Earth, is comprised of an $8 \mathrm{GHz} \mathrm{CW}$ radar. The radar illuminates an area of the Earth's surface, and the signal reflected from the surface is received and demodulated. The motion of the Earth changes the distance the electromagnetic waves travel, resulting in a phase modulation of the received signal that is proportional to the surface displacement. The surface displacement is obtained by demodulating the received signal. A homodyne system is used with in-phase and quadrature mixers to demodulate the received signal. Both the surface displacements due to the seismic waves and the variations in path length associated with the static contours of the ground are obtained separately from the outputs of the two mixers. The two biggest challenges to make the radar perform adequately for the mine detection system are 1) to make it sufficiently sensitive to be able to detect the small vibrations, and 2) to make the spot size (the area on the surface illuminated by the electromagnetic waves) sufficiently small to image the seismic wave field. The radar can measure displacements of 1 $\mathrm{nm}\left(10^{-9} \mathrm{~m}\right)$ as currently configured which is more than sufficient to measure the surface displacements due to the seismic waves that are on the order of $1 \mu \mathrm{m}$. To obtain this sensitivity, the radar was designed to minimize the effects of noise, such as the phase and amplitude noise of the source and electromagnetic interference from low-frequency magnetic fields. The spot size of the radar must be smaller than approximately one half of the shortest wavelength of the seismic waves. Currently, a small spot size is obtained by using an open-ended waveguide as the antenna for the radar. This antenna produces a spot size of about $2 \mathrm{~cm}$ in diameter when the open end of the antenna is placed within a few centimeters of the surface. A field operational mine detection system could contain many of these sensors in a planar 


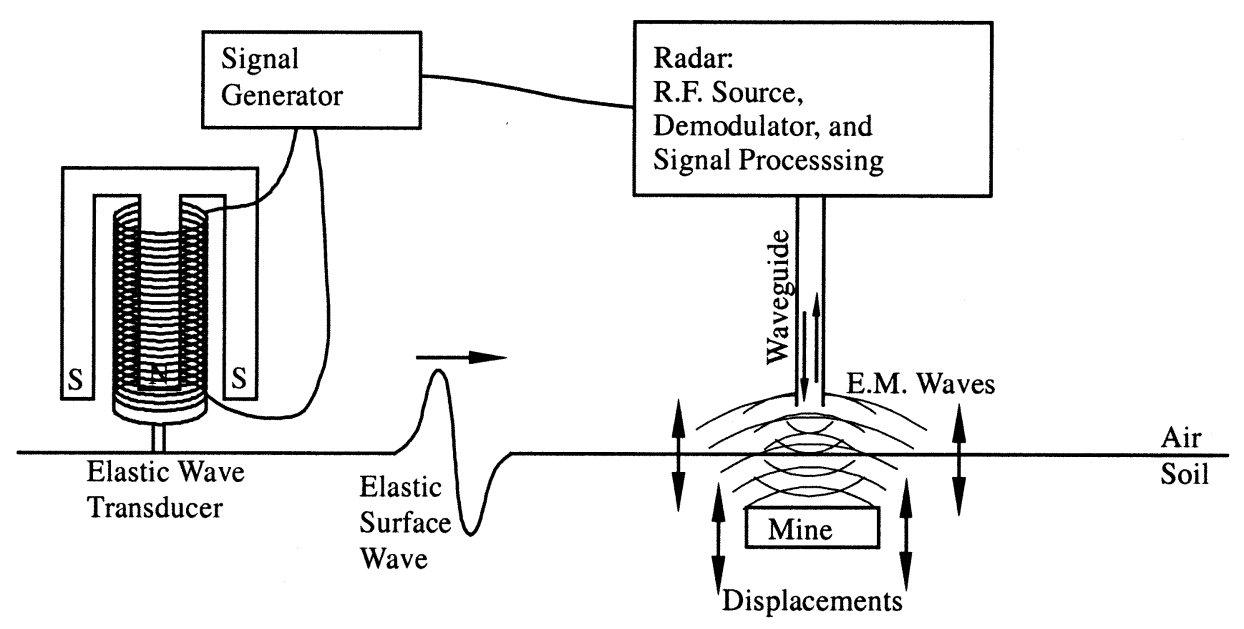

Fig. 2. Configuration of seismic mine detection system.

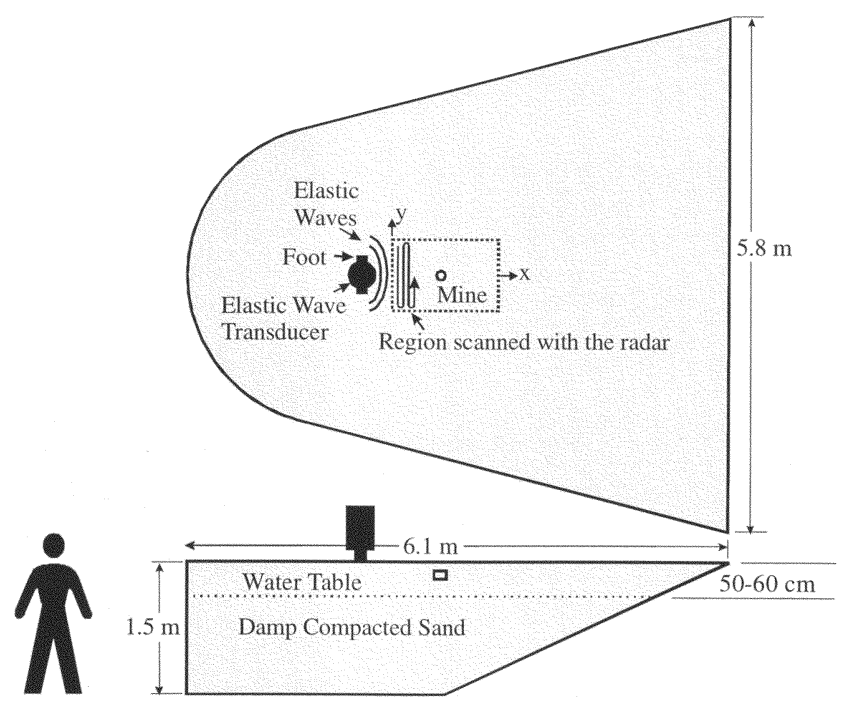

Fig. 3. Experimental soil tank facility.

array. For the laboratory system a single radar sensor is translated above the surface of the earth in order to synthesize this array. ${ }^{1}$

Laboratory testing has been conducted in a wedge-shaped tank, which is depicted in Fig. 3, filled with damp compacted sand to simulate soil. The seismic source is located as indicated in Fig. 3 and is bidirective toward the search area and the back wall. A water table is maintained 50 to $60 \mathrm{~cm}$ below the surface of the tank. Damp compacted sand was chosen as the soil surrogate, because it can be dug up, refilled, and repacked with fairly good repeatability. This repeatability is very important because it allows for comparisons between experiments and thus, easier interpretation of the experiments. The sand surface is periodically re-wet from above and is compacted prior to measurements. This maintains cohesion in the near surface layers of sand

\footnotetext{
${ }^{1}$ The synthetic array is formed on a seismic not an electromagnetic time scale by physically moving, rather than steering, the radar sensor. The seismic wavefronts represented in the data are, therefore, compilations of many distinct seismic events produced by identical source signals at different times (some several hours apart). This form of array synthesis is the reason for the inordinately long scan times associated with the experimental system.
}

that have no bias force created by the weight of overlying material. Surface cohesion is needed to mimic naturally occurring soils. Natural cohesion of particles is due to the water content, weathering, and the presence of fine clays and minerals that cement the soil particles together. Field experiments and published data have verified the realism of compacted damp sand as a soil surrogate because of the comparable wave speeds [3], [6]-[9].

Simulated mines, inert mines, and clutter such as rocks and sticks can be buried within a $2 \mathrm{~m} \times 2 \mathrm{~m}$ scannable region in the center of the tank. The typical scan region, $80 \mathrm{~cm} \times 120 \mathrm{~cm}$, is outlined in Fig. 3. The scan region is sufficiently far from the tank walls that wall reflections can be time-gated out of the data. The sensor can be scanned over this region with a three degree of freedom positioner fixed above the tank.

Experiments in the tank indicate the presence of two measurable propagating wave types: a slow large amplitude surface wave that propagates at 80 to $90 \mathrm{~m} / \mathrm{s}$ and a smaller faster bulk wave which propagates at 190 to $250 \mathrm{~m} / \mathrm{s}$. The faster wave is consistent with previously reported bulk compressional waves in soil and sand [6], [7], and the slower wave matches reported measurements of high-frequency $(>50 \mathrm{~Hz})$ Rayleigh wave speeds [3], [8], [9]. Direct measurement of the compressional wave speed at the surface is difficult because these waves are refracted upwards, making the actual path between the source and receiver uncertain. Direct measurement of a bulk shear wave velocity is not possible at the free surface. This should be slightly faster than the Rayleigh wave speed and can be computed to be 90 to $100 \mathrm{~m} / \mathrm{s}$ from the measured Rayleigh and compressional wave speeds.

Data taken in a one-dimensional (1-D) scan away from the source are depicted in Fig. 4. Here the time waveforms are depicted in a waterfall format or seismogram, where each trace has been offset vertically to represent the spatial separation of the measurement points. This creates a pseudo-surface effect. Individual modes of propagation can be seen as lines of delay connecting wave fronts. The lines on this figure represent subjective evaluations of the phase velocities for the two obvious propagation modes. Dispersion is apparent in the surface wave pulse. Absorption, vertical stratification, inhomogeneity, or nonlinear effects can cause this. Efforts are currently 


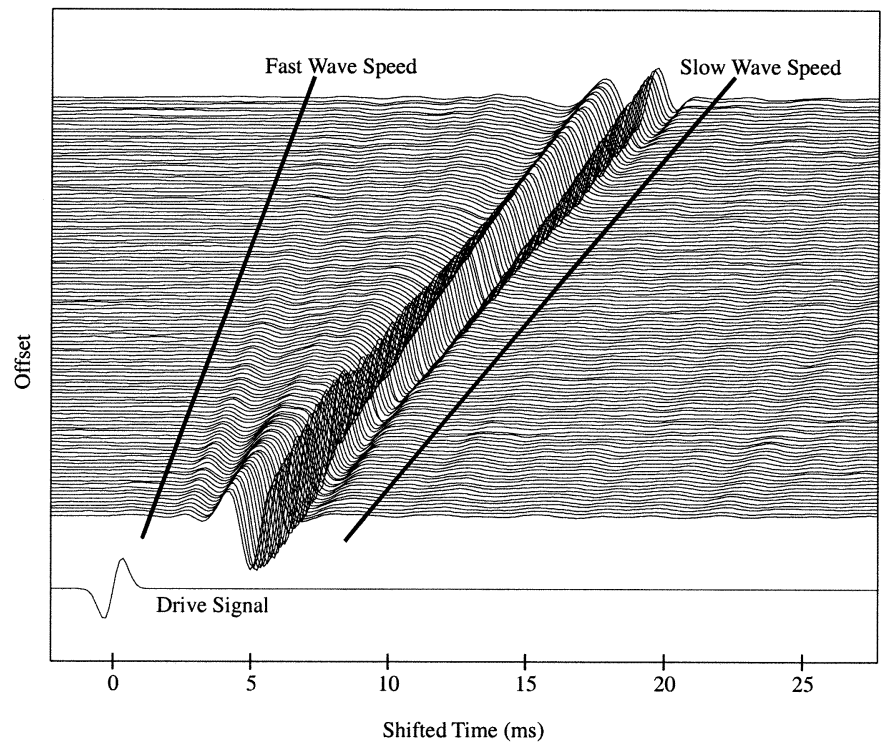

Fig. 4. Seismogram of wave propagation through soil tank measured in $1 \mathrm{~cm}$ increments on a $120 \mathrm{~cm}$ scan.

underway to model and measure each of these contributions. It is also possible that interference with simultaneous arrivals of refracted and reflected bulk waves may create the appearance of dispersion.

Similar data are depicted in Fig. 5 as a pseudo-color graph. Here, the amplitude of the surface displacement at a single instant in time is represented for all measurement locations within the scan region. Out of plane displacement magnitudes are shown on a color scale. The plot area represents the physical dimensions of the surface of the ground. The source is located to the left side of this image, and the incident waves propagate to the right. This convention is used for all the pseudo-color images presented in this paper. The wavefronts apparent in this figure are associated with the Rayleigh wave. At the time instant shown, the compressional wavefronts have propagated beyond the scan region. A sequence of these plots can be used to animate the wave propagation throughout the scan region and visualize interactions with buried objects.

A swept-frequency chirp is currently used to efficiently acquire the data. The sensor is moved to a measurement location, and the source is then driven with a 4-s chirp covering the frequency band of interest. The time record due to the chirp is used to reconstruct the transfer function of the drive signal to the measured displacement [4]. This is then used with a simulated drive signal of shorter duration, with similar bandwidth, to compute a corresponding transient displacement. For the present work, a differentiated Gaussian pulse with a center frequency of $450 \mathrm{~Hz}$ is used as the simulated drive signal. The drive signal is graphed in Fig. 4. The shorter pulse allows for the time separation of different wave types and propagation paths. Similar data have been taken using the shorter pulse as the initial drive signal. The number of record averages required to achieve an SNR comparable to the method using the chirp was time preclusive. However, the data acquired in this way were identical to the compressed waveforms.

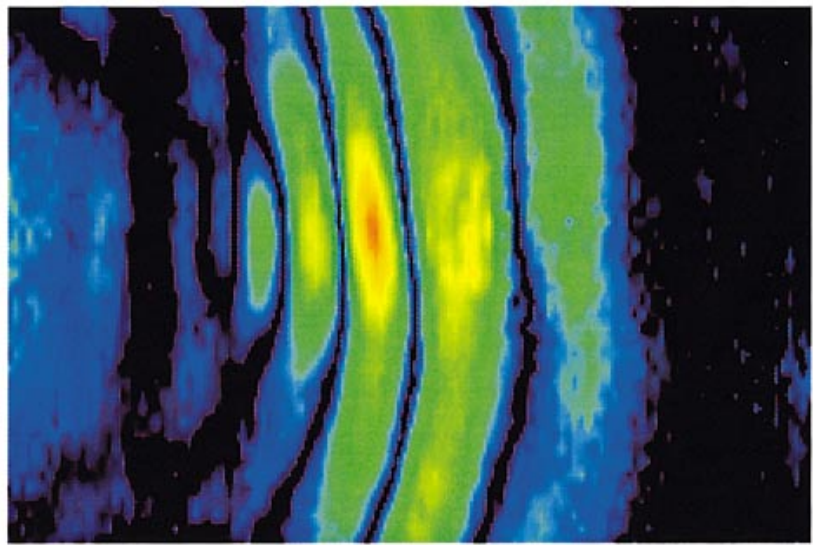

Fig. 5. Transient displacement of the sand surface on a $40 \mathrm{~dB}$ scale at an instant when a Rayleigh wavefront is midway through the $80 \mathrm{~cm} \times 120 \mathrm{~cm}$ measurement region.

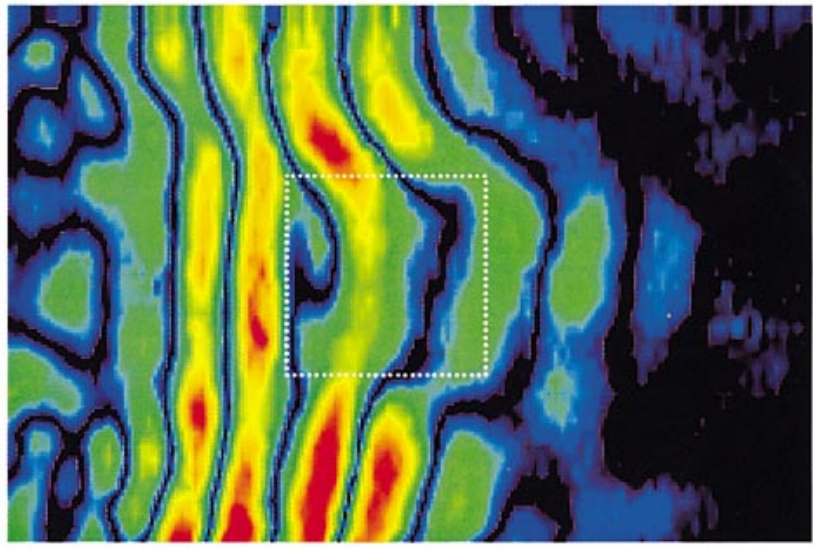

Fig. 6. Interaction of Rayleigh waves with a rigid mine simulant (white outline) buried $5 \mathrm{~cm}$ deep with the surface in an $80 \mathrm{~cm} \times 120 \mathrm{~cm}$ scan region on a $40 \mathrm{~dB}$ scale.

The data acquisition scheme presumes a linear response of the sand and the mine. Sand, however, is a highly nonlinear media. Drive levels that are very low compared with the capabilities of the source are used to prevent nonlinear responses from producing artifacts in the processed data. Most of the observed nonlinearities occur at or near the source where displacements are largest. These nonlinearities significantly limit the amplitude of the waves that can be launched into the sand.

The experimental model has been configured to emphasize data integrity over scanning speed. Currently $9 \mathrm{~h}$ are required for a complete two-dimensional scan of $121 \times 41$ measurement points $\left(\sim 1 \mathrm{~m}^{2}\right)$. As this is clearly inappropriate for a field-operational mine detection system, several techniques are being considered to improve speed without sacrificing measured signal levels. Individually, each of these offers modest time savings, but taken in total, they should reduce scan times to a few seconds per square meter.

Physically arraying the displacement sensor will speed scanning by a factor of the number of elements in the array. If, in lieu of the chirp, an $M$-sequence of similar bandwidth is used, this should allow a $50 \%$ reduction in integration time [11]. Elimination of spatial oversampling with the current system will cut 
measurement times by a factor of four to eight. Improved source design, reduced operating bandwidth, simultaneous processing of data, and an effective scheme for dealing with nonlinear artifacts will provide additional, interrelated improvements.

\section{RigID Mine SimUlants}

Some experiments on seismic mine detection were conducted using mine simulants that did not incorporate mine-like structures. A $30 \mathrm{~cm} \times 30 \mathrm{~cm} \times 7.5 \mathrm{~cm}$ acrylic block was used as an AT mine model representing only the size, shape, and density of an actual target. The primary cue for the identification of this simulant was found to be its combined stiffness and geometric regularity. The simulant responded in a nearly rigid way to the wave motion of the surrounding soil. The motion of the simulant was much like that of a ship at sea. Incident wave fronts appeared to break at the leading edge of the mine; waves were shed behind the mine prior to their arrival via the diffracted path around the mine, and the mine itself rocked about a point roughly $1 / 3$ of the way along its length. Fig. 6 shows a pseudo-color graph of the displacement of the measurement surface above the acrylic mine buried $5 \mathrm{~cm}$ below the surface at an instant shortly after the Rayleigh wave has reached the mine location. The wavefronts to the right of the mine are seen to be curved, because of the accelerated arrival of the waves shed by the mine. The mine itself is moving less than the surrounding sand, and the edges of the mine are apparent as discontinuities in the surface displacement. Significant dispersion is also observed in a wave that propagates in the layer of soil over the mine. This layer forms a waveguide between the mine and the free surface. Although this sort of mine simulant has been imaged, it would be difficult to distinguish from buried clutter. The feature that makes this mine distinctive from rigid clutter such as rocks is its geometric regularity. Accurately outlining the shape of the simulant required wavelengths that would not penetrate to the full depth range expected for a mine of this size. ${ }^{2}$

\section{RESONANT MinES}

Although it was possible to image mine simulants without representative structural details, the dominant features observed in the signatures of inert AP and AT mines were found to be soil-loaded resonances of the mine case and trigger mechanism [12], [13]. These are excited by the passage of the Rayleigh wave and characterized by large displacements that persist after the passage of the incident pulse. Numerical models indicate that the resonance is due to flexural waves excited in the case/trigger of the mine and in the layer of soil above the mine [2]. The mine trigger is comprised of plastic that is much stiffer than the overlying sand. However, this plastic is thin in comparison to the soil layer. The stiffness and the mass of both the mine trigger and the overlaying layer of soil are believed to be important contributors to the resonance.

Although mines exhibiting resonances scatter a larger propagating wave field than similarly sized nonresonant objects, the

\footnotetext{
${ }^{2}$ This is a different problem from the competing operational frequency requirements encountered with the pulse-echo scheme previously discussed. Here, the competition between frequency, mine size, and depth pertains to target classification rather than detection.
}

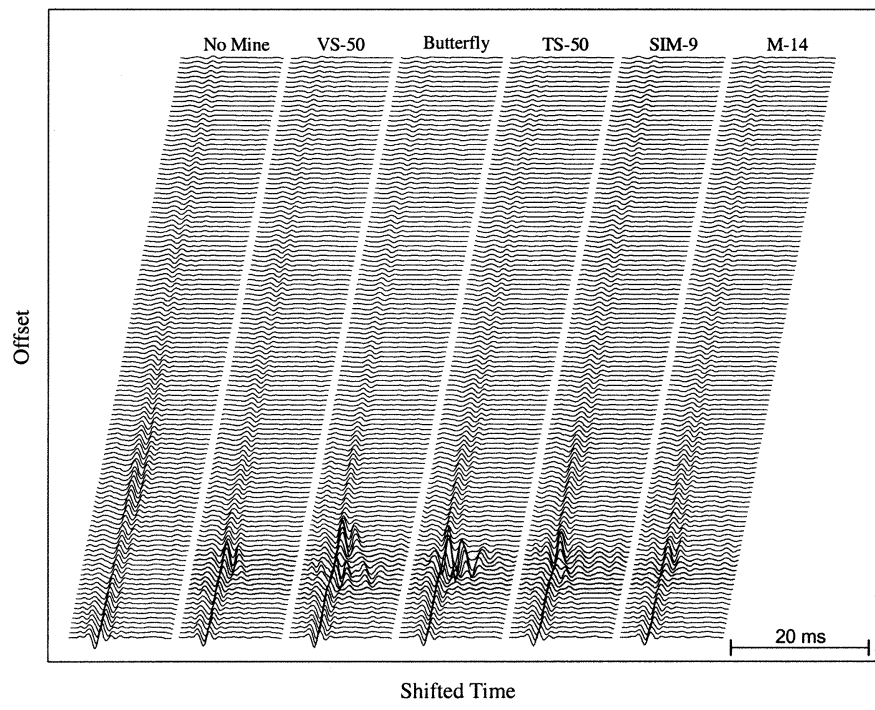

Fig. 7. Displacement of the surface of the sand above various AP mines, measured in $1 \mathrm{~cm}$ increments on a $120 \mathrm{~cm}$ scan. All AP mines were buried 0.6 $\mathrm{cm}$ deep.

most pronounced feature of the field scattered by these mines is its primarily local nature resulting from a dominantly reactive soil loading. For the mine types studied thus far, the localized resonant motion has been an excellent indicator of a mine's location and extent. Fig. 7 shows a seismogram for each of five different AP mine types compared to the no-mine case. Four of these can be seen in the photograph in Fig. 10. The waveforms have been windowed so that only information around the arrival time of the incident Rayleigh wave pulse is shown. To varying degrees, a resonance is apparent for each AP mine type. These mines were buried $0.6 \mathrm{~cm}$ below the surface.

2 -D scans over buried inert mines show the mine resonance features more clearly. In Fig. 8, surface displacement magnitudes are represented over the scan region for three instants in time when an inert TS-50 mine is buried $1.3 \mathrm{~cm}$ deep in the scan region. At first (a), the incident wave has not reached the mine. In the second instant (b), the wave front has reached the mine and can be seen to produce large displacements in the soil layer above the mine. In the third instant (c), the incident signal has propagated beyond the mine. However, there is still ringing at the mine, and it is shedding energy by radiating small amplitude wave fronts.

Imaging of mines from the surface displacement measurements can be done in many ways. The current technique, which has been outlined in a previous paper [14], involves a multistep process. The 2-D scan data are filtered in the wavenumber domain to remove all components propagating away from the source, leaving the reflected waves and a portion of the nonpropagating waves. The remaining information is windowed in time around the arrival time of the incident signal and averaged to form a pixel in the final image. Fig. 9 shows an image formed in this way from the data that was used to generate the frames of Fig. 8.

\section{Buried Clutter AND MultiPle Mines}

Many objects, which are mine-sized or larger and have elastic properties quite different from soils, are buried at shallow depths 

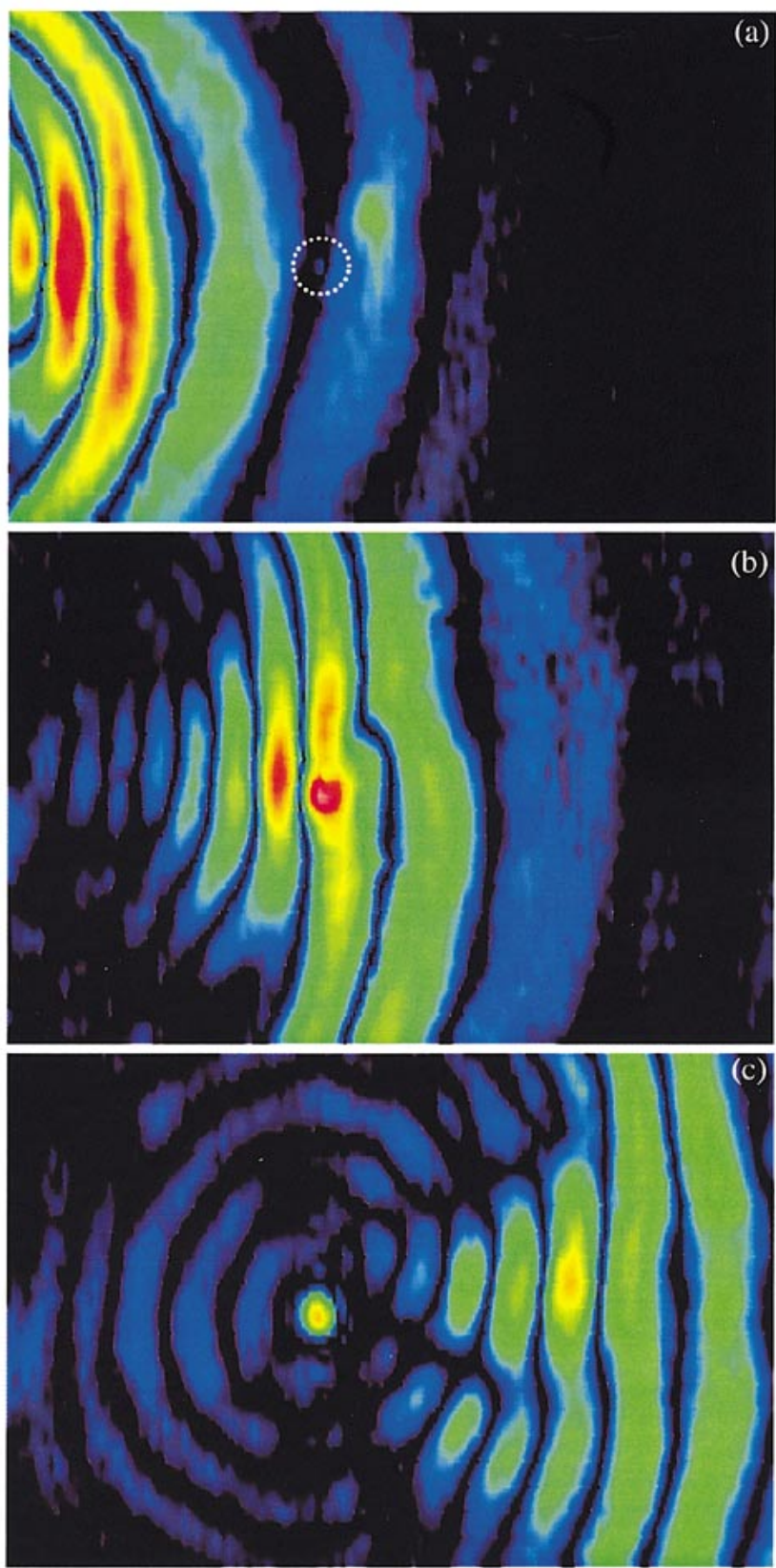

Fig. 8. Sand surface displacements in an $80 \mathrm{~cm} \times 120 \mathrm{~cm}$ scan region at three instants on a $40 \mathrm{~dB}$ scale (a) before wavefronts reach a TS-50 AP mine (outlined) buried $1.3 \mathrm{~cm}$ deep at $t=3.0 \mathrm{~ms}$, (b) during interaction of the waves with the mine at $t=6.9 \mathrm{~ms}$, and (c) after the wavefronts have passed the mine at $t=$ $12.2 \mathrm{~ms}$.

in the ground. Rocks and tree roots are good examples of this sort of clutter. For a mine detection system to be effective, it must permit imaging that accurately depicts the size and location of a mine and distinguishes it from this type of clutter.

A common practice of mine warfare is to plant multiple AP mines in close proximity to AT mines. The AP mines thereby protect the AT mine from sappers who can more easily detect the larger object and remove it with little personal danger. This poses a unique detection problem in that it requires a system to operate with sensitivity appropriate to both mine types simultaneously. Also, the system must be capable of distinguishing

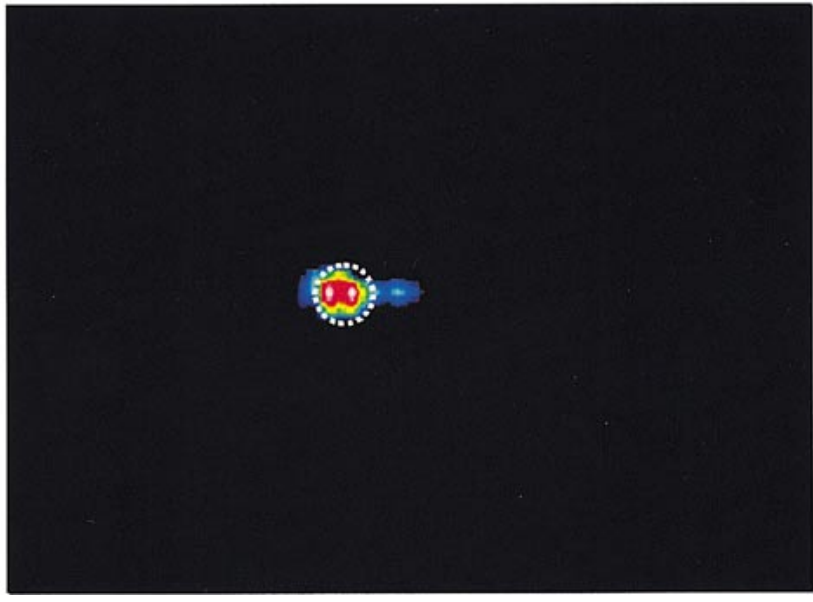

Fig. 9. Image formed of a single TS-50 AP mine (outlined) buried $1.3 \mathrm{~cm}$ deep in an $80 \mathrm{~cm} \times 120 \mathrm{~cm}$ scan region on a $25 \mathrm{~dB}$ scale.

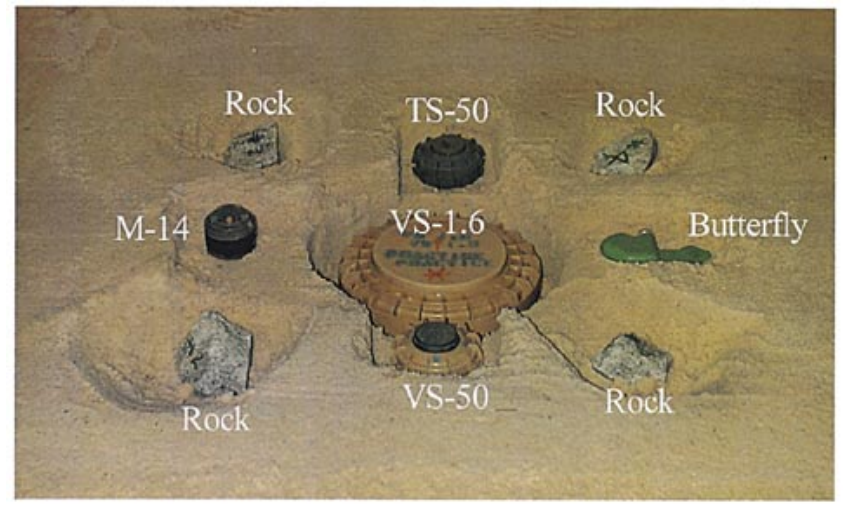

Fig. 10. VS-1.6 AT mine surrounded by TS-50, butterfly, VS-50, and M-14 AP mines and rocks. The burial depths for the mines were $4.5 \mathrm{~cm}$ for the VS-1.6; $2 \mathrm{~cm}$ for the TS-50, VS-50, and butterfly; and $0.5 \mathrm{~cm}$ for the M-14. The burial depths for the rocks were $3.5 \mathrm{~cm}, 1.5 \mathrm{~cm}, 2 \mathrm{~cm}$, and $1 \mathrm{~cm}$ (clockwise, starting with the upper left rock).

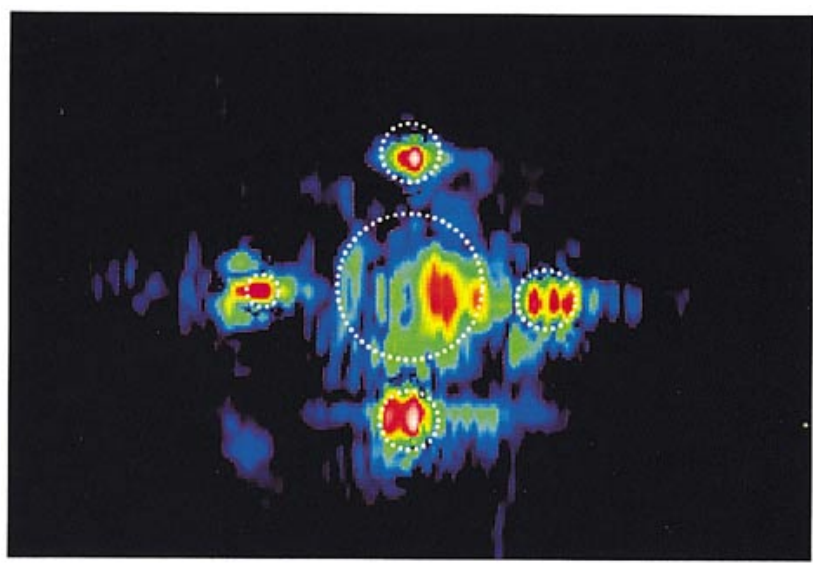

Fig. 11. Image of AT mine (outlined) and surrounding AP mines (outlined) in an $80 \mathrm{~cm} \times 120 \mathrm{~cm}$ scan area on a $30 \mathrm{~dB}$ scale.

individual targets and rejecting ghost images formed by multiple scattering effects.

An experiment was performed to test the effects of buried clutter and multiple mines. In this experiment, an inert VS-1.6 


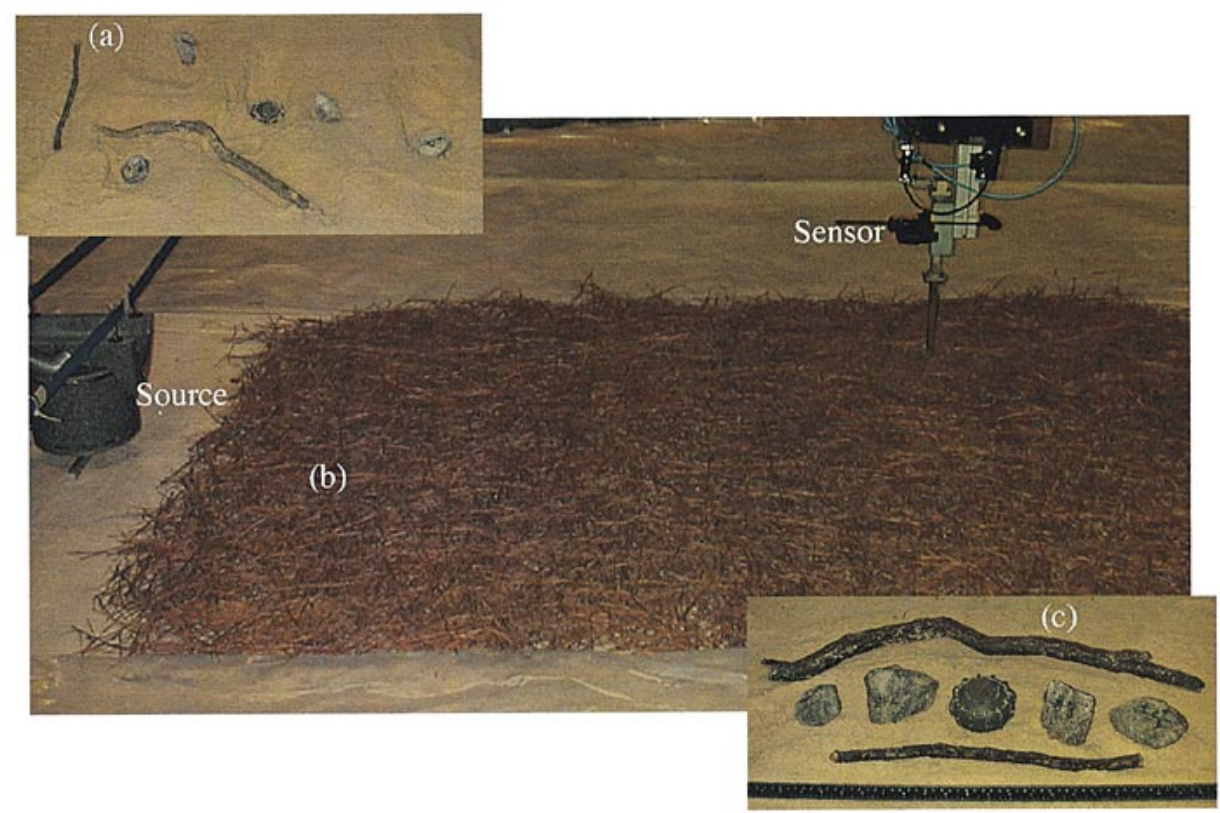

Fig. 12. Simulated minefield (a) relative locations of TS-50 AP mine and clutter objects (b) pine straw surface covering (c) relative scale of buried objects.

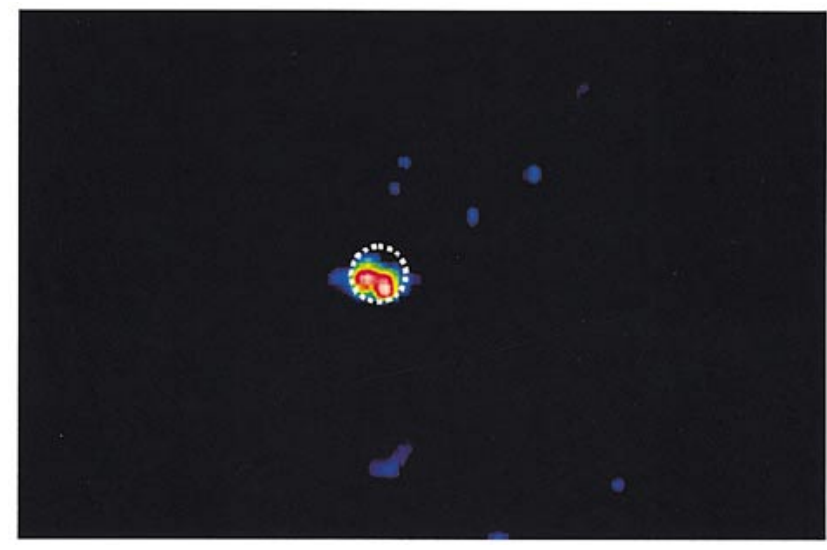

Fig. 13. Image formed of TS-50 AP mine (outlined) and clutter below pine straw surface cover in an $80 \mathrm{~cm} \times 120 \mathrm{~cm}$ scan region on a $25 \mathrm{~dB}$ scale.

AT mine was surrounded by four different inert AP mines and four mine-sized rocks. The arrangement of this burial and relative scale of the objects can be seen in Fig. 10.

The image formed from the data taken over the multiple mine burial is depicted in Fig. 11. The number of mines present and their relative locations have been accurately imaged. The image of the AT mine is seen to be strongest at the back edge. This is due to the reflection at the back edge being stronger than that at the front. The effects of the rocks are much smaller than those of any of the mines. The largest rock is barely discernible on the $30-\mathrm{dB}$ dynamic range used to generate the image. The reason for this is that the rocks do not exhibit resonances within the frequency range of the incident signal. This result has been modeled numerically with a 3-D finite-difference time-domain (FDTD) model [15]. The FDTD model incorporates measured material properties and approximations to the complex geometry of the rocks and mine. Results from the FDTD model are in good agreement with the experimental measurements.

\section{GROUND COVER}

In early testing of the seismic mine detection system, the surface of the soil surrogate used in the experiments was maintained level, smooth, and bare. This provided the radar sensor with a seismically modulated EM reflection from the surface that was nearly optimal (large and uncorrupted). In the field, the surface will be rough and lie under some type of ground cover. A sensor that sees through common surface cover such as grass or light vegetation is essential for practical system operation. Some types of ground cover, obviously, will be opaque to the radar's interrogation signal. These will require special consideration and possibly an alternate sensor design. Standing water is an example of such a problematic case. In general, vegetation is likely to be translucent to the radar sensor with a portion of the EM reflection originating in the vegetation and the remainder coming from the surface below. The signal that is reflected from the vegetation can corrupt the total return in two ways. First, it will reduce the relative contribution of the electromagnetic signal component reflected from the surface. This will reduce the level of the measured displacement signal. Second, any motion of the vegetation will produce an additional modulation of the carrier unrelated to the motion of the underlying surface. This will increase the effective noise floor.

To test the ability to penetrate surface cover, pine straw was selected both for the convenience of its application and because it is a commonly occurring ground covering. In the experiment, a $2.3 \mathrm{~cm}$ layer of pine straw was spread over the model's surface beneath which a TS-50 AP mine was buried $1.3 \mathrm{~cm}$ deep along with 4 mine sized rocks and two sticks at similar depths. The layout of this experiment can be seen in Fig. 12 .

Fig. 13 shows the image formed from the pine straw covered surface. The location and extent of the TS-50 mine are apparent. There is less contrast in this image than for similar images formed in the absence of surface covering. An examination of the signals that contribute to the image reveals that the 


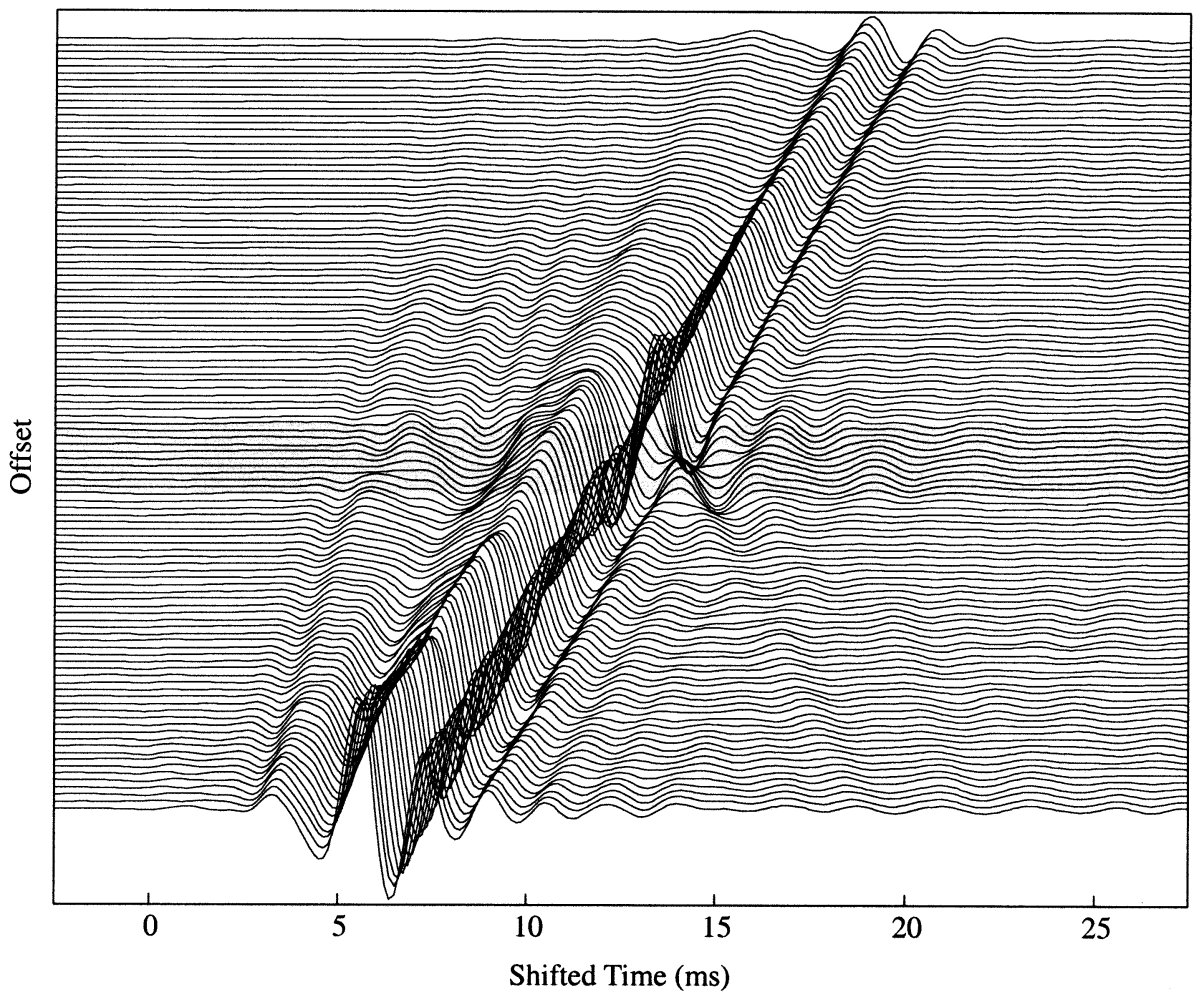

(a)

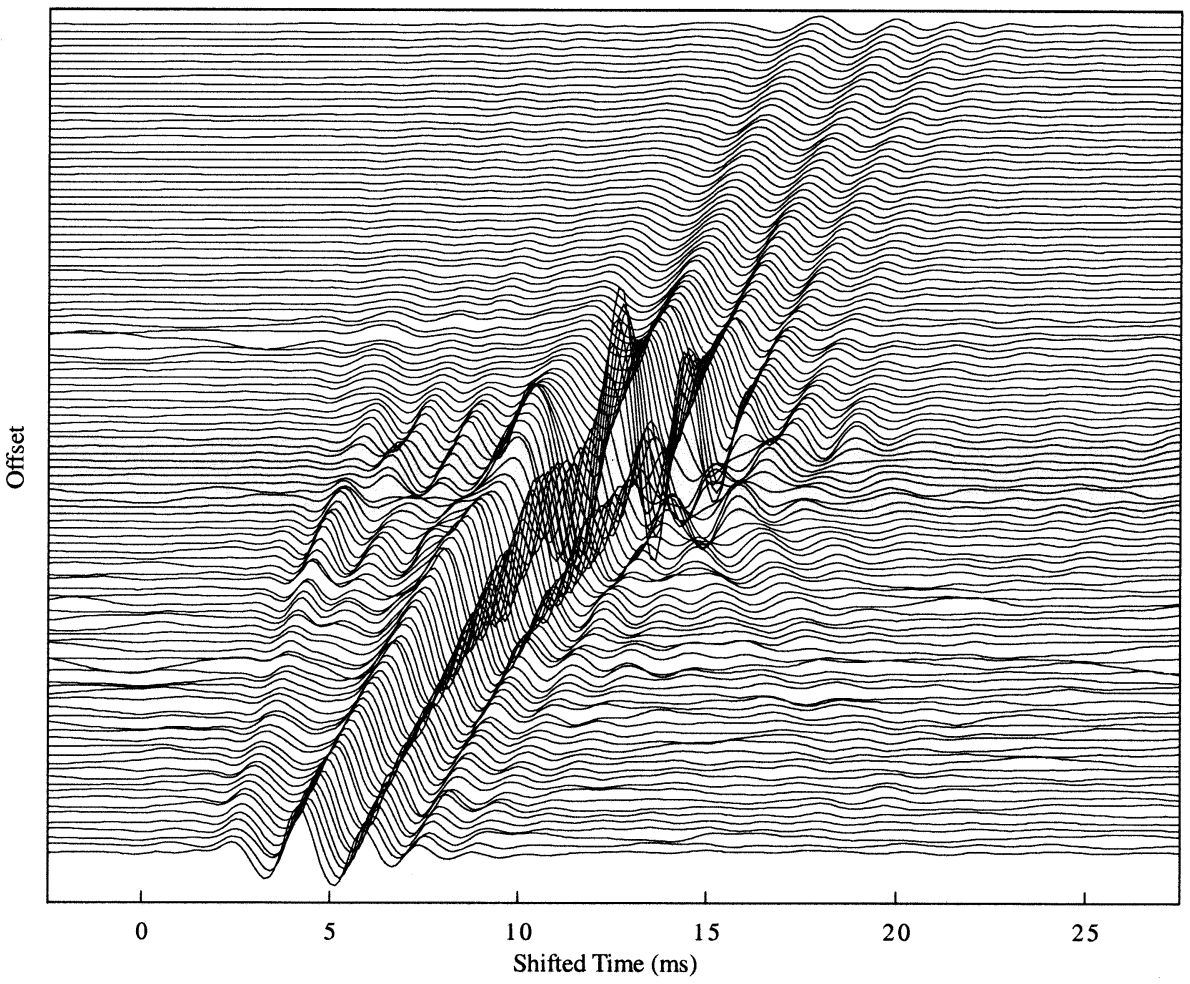

(b)

Fig. 14. Seismograms showing surface displacements measured in $1 \mathrm{~cm}$ increments on a $110 \mathrm{~cm}$ scan across a compliant AP mine simulant buried $4 \mathrm{~cm}$ deep (a) with the surrounding sand uniformly compacted and (b) with the sand locally disturbed around the mine.

dominant effect of the pine straw was to force an increase in the separation between the antenna and the ground's surface that resulted in a reduced signal level and a loss of some spatial resolution. The reduced signal level causes an increase in the noise floor that reduces the contrast in the image.

\section{TRENCHING EFFECTS}

It is well known that it is often easier to detect the recently disturbed earth around a buried mine than to detect the mine itself. This burial disturbance is called trenching. Observations 
of trenching effects suggested an experiment to test the impact of recent burial on the detectability of an AP mine. When a mine has been recently buried, the soil around it is likely to be less compacted than in its greater surroundings. This is not surprising since an individual placing a mine is apt to be hesitant to compact the soil around it. Over time, compaction will occur due to weathering. The trenching effect is, therefore, a poor cue for reliable mine detection. A local lack of cohesion is not the primary detection cue under investigation with the current system. It was suspected that trenching could degrade system performance by decoupling the mine from the surrounding soil through which the seismic excitation is applied.

Fig. 14 shows seismograms generate 1-D scans over a resonant AP mine simulant buried $4 \mathrm{~cm}$ deep. The mine simulant was a hollow plastic case with thin walls approximately $9 \mathrm{~cm}$ in diameter and $2.2 \mathrm{~cm}$ in height. In the upper plot, the sand in the entire scan region was tilled and recompacted after the mine burial. In the lower plot, only the sand immediately around the mine was disturbed for the burial creating a local inhomogeneity. The resonance of the mine simulant is apparent in both images, but is much more pronounced in the presence of the local inhomogeneity. This surprising result is believed to be attributable to the hole focusing the incident wave to the location of the mine. The hole also reduces the radiation damping of the mine's resonance because of the poor match to the properties of its surroundings. These effects have been reproduced with numerical models that represent the filled hole as a cylinder of material having $20 \%$ lower wave speeds than the bulk of the medium. Both the experimental model and the numerical model predict that the trenching effect enhances the resonant response of the mine and thereby improves detection performance [16].

\section{DEPTh RANGE OF DETECTABILITY}

There are two ranges of mine size and burial depth that are of concern for the mine detection problem. The first of these is for AP mines that are typically a few hundred milliliters in volume and buried such that the mine trigger is a few $\mathrm{cm}$ below the soil surface. AT mines are typically several liters in volume and have burial depths up to a few decimeters. Rayleigh waves penetrate the surface to a depth proportional to their wavelength. It is therefore likely that AT mine detection will dictate the low frequency requirements of the detection system. Since the primary mine detection cue that is exploited with the current system is a resonance of the mine, it is unlikely that the AT mine problem is a simple direct scaling of the AP mine imaging technique. It must, therefore, be specifically addressed. Currently, the operating band of the system $(100-2000 \mathrm{~Hz})$ is dictated by the design of the experimental model. Source response, ambient noise, and reverberation impose the low-frequency limit. Attenuation in the soil surrogate limits the operating bandwidth at high frequencies.

A VS-1.6 AT mine was examined as a function of burial depth using a 1-D scan. The results for this are depicted in the seismograms shown in Fig. 15. The seismograms shown in Fig. 15(a) have been windowed in the same manner as those in Fig. 7. In

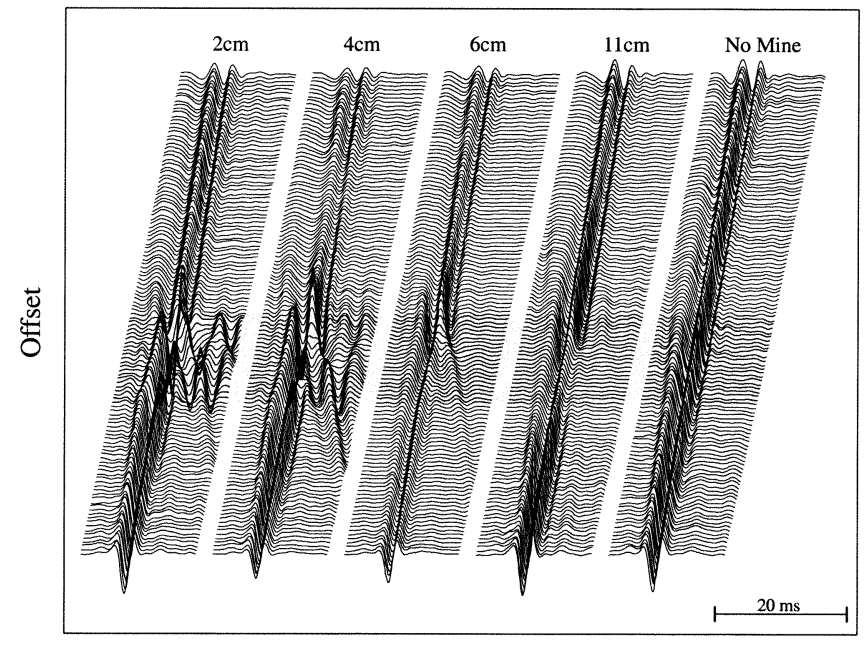

Shifted Time

(a)

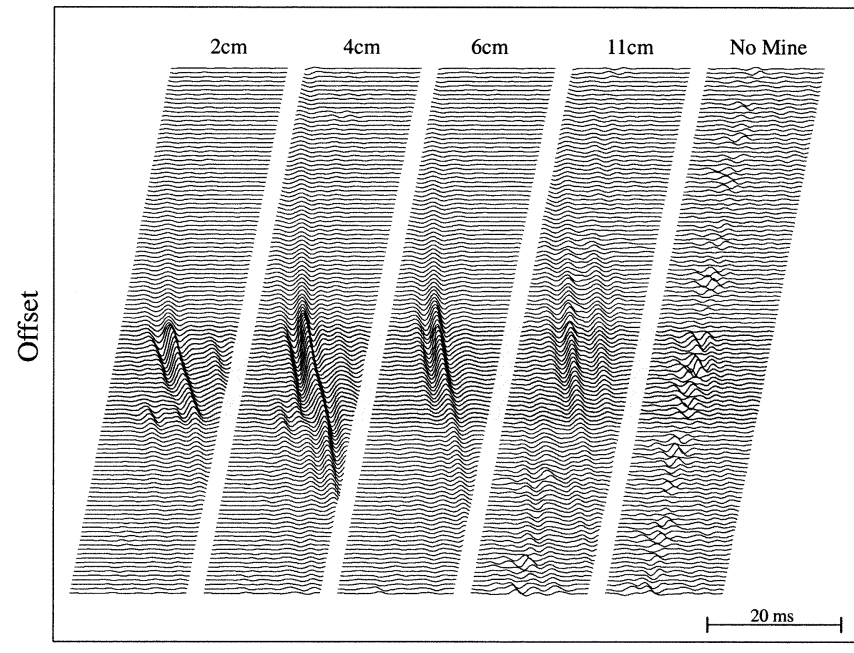

Shifted Time

(b)

Fig. 15. Windowed seismograms of VS-1.6 AT mine at various depths, measured in $1 \mathrm{~cm}$ steps on a $120 \mathrm{~cm}$ scan. (a) Raw data and (b) forward propagating waves filtered out.

Fig. 15(b), the forward components of the wave field have been filtered out to emphasize the effects of the mine. The ripples seen in the no-mine graph in Fig. 15(b) are due to imperfect filtering of the incident wave. The root mean square (RMS) value of each trace shown in Fig. 15(b) would represent a pixel along the centerline if an image were formed.

Although the raw data covered the same band as in the previous seismograms, it was processed in a slightly different way. The measured transfer functions were convolved with a pulse shape that had a center frequency that was an octave lower (225 $\mathrm{Hz}$ as opposed to $450 \mathrm{~Hz}$ for the AP mine scans). The experimental data used to reconstruct either pulse response are identical. The difference is in the postprocessing. Many such processing techniques could be used simultaneously to interrogate for specific targets or burial depth ranges. The pulse with the lower center frequency was chosen for the AT mine in order to emphasize the effects of the spectral components which penetrated the soil to a sufficient depth to interact with the mine. 
From data in Fig. 15 the presence of the mine is apparent up to a burial depth of about $11 \mathrm{~cm}$. Above the mine, an amplification of the incident wave can be observed for 2,4 , and $6 \mathrm{~cm}$ burial depths. Waves that have been reflected from the mines are clearly seen propagating away for these burial depths. The strength of these waves for the 2 and $4 \mathrm{~cm}$ burial depths appears to be inversely related to the duration of the localized motion above the mine. This indicates that the radiation of Rayleigh waves constitutes a significant source of damping for the mine motion. There is also evidence of dispersion in the soil layer above the mine. The leading edge of the incident signal is clearly delayed as it passes above the mine. Also, the pulse shape and arrival time well beyond the mine are quite different than they are at the same location for the no-mine case.

\section{CONCLUSIONS}

Detection of simulated and inert AP and AT mines using Rayleigh seismic waves and a radar-based noncontact displacement sensor has been demonstrated with a laboratory experimental model. Scenarios that mimic a variety of realistic field conditions have been modeled. The system has proven to be effective in these scenarios. Modeled conditions include the presence of natural surface covering, buried clutter, multiple mine burials, trenching, and deeply buried mines. Experimental system performance shows good agreement with published data and with analytical and numerical models of seismic propagation.

\section{REFERENCES}

[1] D. L. Patel, "Handbook of land mines and military explosives for countermine exploitation," Tech. Rep. 2495, U.S. Army Belvoir Res. Devel. Center, Mar. 1992.

[2] C. T. Schroeder and W. R. Scott Jr., "A finite-difference model for elastic waves in the ground," IEEE Trans. Geosci. Remote Sensing, vol. 38, pp. $1505-1512$, July 2000.

[3] "Feasibility of acoustic landmine detection," Tech. Rep. 7677, BBN Syst. Technol., Cambridge, MA, May 1992.

[4] C. Stewart, "Summary of mine detection research," Tech. Rep. 1636-TR, U.S. Army Eng. Res. Devel. Labs, Corps Eng., Belvoir, VA, vol. I, May 1960.

[5] M. C. Junger and D. Feit, Sound, Structures, and Their Interaction: Acoust. Soc. Amer., 1993, pp. 31-34 and 60-64.

[6] J. M. Sabatier, H. E. Bass, L. N. Bolen, and K. Attenborough, "Acoustically induced seismic waves," J. Acoust. Soc. Amer, vol. 80, pp. 646-649, Aug. 1986.

[7] R. Bachrach, A. Nur, and J. Dvorkin, "High-resolution shallow-seismic experiments in sand, Parts I and II," Geophysics, vol. 63, no. 4, pp. 1225-40, 1998.

[8] P. Smith, P. S. Wilson, F. W. Bacon, J. F. Manning, J. A. Behrens, and T. G. Muir, "Measurement and localization of interface wave reflections from a buried target," J. Acoust. Soc. Amer., vol. 103, pp. 2333-2343, May 1998.

[9] M. Westebbe, J. F. Bohme, H. Krummel, and M. B. Matthews, "Model fitting and testing in near surface seismics using maximum likelihood in frequency domain," in Conf. Record 32d Asilomar Conf. Signals, Systems and Computers, Pacific Grove, CA, Nov. 1998.

[10] G. Godfrey, Perturbation Signals for System Identification. Englewood Cliffs, NJ: Prentice-Hall, 1993.

[11] A. Behboodian, W. R. Scott, Jr., and J. H. McClellan, "Relative performance of different perturbation signals for measuring the impulse response of linear time-invariant systems," IEEE Trans. Instrum. Meas., to be published
[12] W. R. Scott Jr., G. D. Larson, and J. S. Martin, "Simultaneous use of elastic and electromagnetic waves for the detection of buried land mines," in Proc. SPIE: 2000 Annu. Int. Symp. Aerospace/Defense Sensing, Simulation, and Controls, Orlando, FL, Apr. 2000.

[13] W. R. Scott Jr., C. Schroeder, and J. S. Martin, "A hybrid acoustic/electromagnetic technique for locating land mines," in Proc. 1998 Int. Geoscience and Remote Sensing Symp., Seattle, WA, July 1998.

[14] A. Behboodian, W. R. Scott Jr., and J. H. McClellan, "Signal processing of elastic surface waves for localizing buried land mines," in Proc. 33rd Assilomar Conf. Signals, Systems, and Computers, Pacific Grove, CA, Oct. 1999.

[15] C. T. Schroeder and W. R. Scott Jr., "Three-dimensional finite-difference time-domain model for interaction of elastic waves with buried land mines," in Proc. SPIE: 2000 Annu. Int. Symp. Aerospace/Defense Sensing, Simulation, and Controls, Orlando, FL, Apr. 2000.

[16] W. R. Scott Jr., C. T. Schroeder, J. S. Martin, and G. D. Larson, "Investigation of a technique that uses both elastic and electromagnetic waves to detect buried land mines," in AP2000 Millennium Conf. Antennas and Propagation, Davos, Switzerland, Apr. 2000.

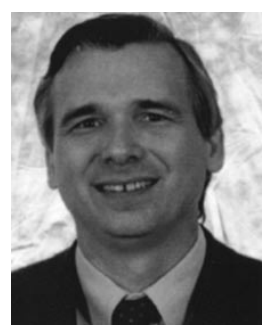

Waymond R. Scott, Jr. (M'00) was born in Calhoun, GA, on April 6, 1958. He received the B.E.E., M.S.E.E., and Ph.D. degrees from the Georgia Institute of Technology (Georgia Tech), Atlanta, in 1980,1982 , and 1985 , respectively.

From 1979 to 1980, he was a Student Assistant and Graduate Research Assistant with the Georgia Tech Research Institute, and from 1980 to 1985, he was a Graduate Research Assistant with the School of Electrical Engineering at the Georgia Institute of Technology, where he is currently a Professor of Electrical and Computer Engineering. His research interests include methods for detecting buried objects using both electromagnetic and acoustic waves, measurement of the electromagnetic properties of materials, transient electromagnetic fields, and numerical methods including the finite element and the finite-difference time-domain techniques.

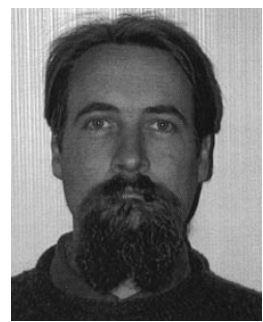

James S. Martin was born in New Haven, CT, on May 6, 1964. He received the B.M.E. and M.S.M.E. degrees from the Georgia Institute of Technology (Georgia Tech), Atlanta, in 1989 and 1994 , respectively, where he is currently pursuing the Ph.D. degree.

He has been working as a Research Engineer in the Woodruff School of Mechanical Engineering, Georgia Tech, since 1989. His research interests include acoustic transducer design, structural acoustics, acoustical oceanography, bioacoustics, and seismology. He is a registered Professional Engineer in the state of Georgia. Mr. Martin is a member of the Acoustical Society of America.

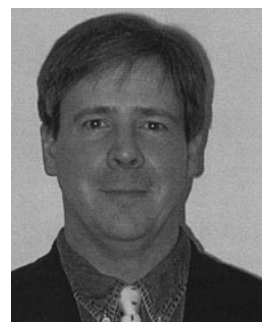

Gregg D. Larson was born in Tulsa, OK, on August 11,1966 . He received the B.E. degree from Vanderbilt University, Nashville, TN, in 1988, and the M.S.M.E. and Ph.D. degrees from the Georgia Institute of Technology (Georgia Tech), Atlanta, in 1990 and 1996, respectively.

From 1990 to 1996, he was a Graduate Research Assistant and Graduate Teaching Assistant with the Woodruff School of Mechanical Engineering, Georgia Tech. Since 1996, he has been a Research Engineer II in the Woodruff School of Mechanical Engineering. His research interests include acoustic transduction, bioacoustics, active vibration control, smart materials, and seismic acoustics.

Dr. Larson is a member of the Acoustical Society of America and the American Society of Mechanical Engineers. 